\title{
Heavy Metals Accumulation Affects Bone Microarchitecture in Osteoporotic Patients
}

\author{
Manuel Scimeca, ${ }^{1,2^{*}}$ Maurizio Feola, ${ }^{3^{*}}$ Lorenzo Romano, ${ }^{3}$ Cecilia Rao, ${ }^{3}$ Elena Gasbarra, ${ }^{3}$ \\ Elena Bonanno, ${ }^{2,4}$ Maria Luisa Brandi, ${ }^{5}$ Umberto Tarantino ${ }^{3}$ \\ ${ }^{1}$ Department of Spatial Biomedicine, Italian Space Agency (ASI), via Del Politecnico Snc, \\ Rome 00133 \\ ${ }^{2}$ Anatomic Pathology Section, Department of Biomedicine and Prevention, University of Rome \\ "Tor Vergata,", via Montpellier 1, Rome 00133, Italy \\ 3Department of Orthopedics and Traumatology, "Tor Vergata" University of Rome," "Policlinico \\ Tor Vergata" Foundation, Viale Oxford 1, Rome 00133, Italy \\ ${ }^{4}$ TMALab S.R.L., Spin-off of University of Tor Vergata, Department of Biomedicine and \\ Prevenction, Rome, Italy \\ ${ }^{5}$ Department of Surgery and Translational Medicine, University of Florence, Florence 50139, \\ Italy
}

Received 21 April 2016; revised 6 July 2016; accepted 7 July 2016

\begin{abstract}
Bone metabolism is affected by mechanical, genetic, and environmental factors and plays a major role in osteoporosis. Nevertheless, the influence of environmental pollution on the occurrence of osteoporosis is still unclear and controversial. In this context, heavy metals are the most important pollutants capable to affect bone mass. The aim of this study was to investigate whether heavy metals accumulation in bone tissues could be related to the altered bone metabolism and architecture of osteoporotic patients. To this end, we analyzed 25 bone head biopsies osteoporotic patients and 25 bone head biopsies of osteoarthritic patients. Moreover we enrolled 15 patients underwent hip arthroplasty for high-energy hip fracture or osteonecrosis of the femoral head as a control group. Bone head biopsies were studied by BioQuant-osteo software, scanning electron microscopy and Energy Dispersive X-ray microanalysis. We found a prevalence of lead, cadmium and chromium accumulation in osteoporotic patients. Noteworthy, high levels of sclerostin, detected by immunohistochemistry, correlate with the accumulation of heavy metal found in the bone of osteoporotic patients, suggesting a molecular link between heavy metal accumulation and bone metabolism impairment. In conclusion, the presence of heavy metals into bone shed new light on the comprehension of the pathogenesis of osteoporosis since these elements could play a non redundant role in the development of osteoporosis at cellular/molecular and epigenetic level. Nevertheless, in vivo and in vitro studies need to better elucidate the molecular
\end{abstract}

Correspondence to: U. Tarantino; e-mail address: umberto.tarantino@uniroma2.it

*These authors contributed equally to this work.

Disclosure statement: Manuel Scimeca, Maurizio Feola, Lorenzo Romano, Cecilia Rao, Elena Gasbarra, Elena Bonanno, Maria Luisa Brandi, and Umberto Tarantino declare that they have no conflict of interest. The study is original and the manuscript has not been published yet and is not being considered for publication elsewhere in any language either integrally or partially except as an abstract. All authors have agreed with the submission in its present (and subsequent) forms.

Contract grant sponsor: ASI (Italian Space Agency); University of Rome “Tor Vergata” (Spatial Biomedicine Center)

Published online 00 Month 2016 in Wiley Online Library (wileyonlinelibrary.com). DOI: 10.1002/tox.22327 
mechanism in which heavy metals can participate to osteoporosis. (c) 2016 Wiley Periodicals, Inc. Environ Toxicol 00: 000-000, 2016.

Keywords: heavy metals; osteoporosis; osteoarthrosis; bone health; environmental pollution

\section{INTRODUCTION}

Osteoporosis is the most common metabolic bone disease, characterized by a decrease of bone mass and a degenerative change of bone microstructure, resulting in an increased risk of fracture (Cerocchi et al., 2013). A rapidly expanding amount of evidence indicates that osteoporosis is likely to be caused by complex interactions between local and systemic regulators of bone cell function. Indeed, osteoporosis results in a dysfunction of physiological bone turnover where endocrine and/or autocrine/paracrine factors negatively affect peak bone mass and/or skeletal homeostasis (Drake et al., 2015, O'Flaherty, 2000). Patients with osteoporosis show a higher propensity to spine, femur and fractures. Osteoporotic fracture caused by a minor injury often occurs in cancellous bone-rich zone, with increasing disability and lethality rates, which may seriously affect the quality of life of older persons (Andreoli et al., 2011).

Several studies, revealed that heavy metals exposure may affect bone mass (James and Meliker, 2013; Sadeghi et al., 2014; Tsai et al., 2015). In particular, lead ( $\mathrm{Pb})$ is associated to bone mass deficiency and osteoporotic condition. $\mathrm{Pb}$ accumulates in bones already during fetal development, and adverse effects on bone metabolism present even after low level of exposure (Berglund et al., 2000; Khalil et al., 2008). It causes growth retardation by inhibiting endochondral ossification. Increased bone turnover and reduced mineralization combine to decrease bone mineral density and mass, and, in the most severe cases, cause osteoporosis (Dermience et al., 2015). Other important heavy metals that influence bone metabolism are cadmium $(\mathrm{Cd})$, copper $(\mathrm{Cu})$, chromium $(\mathrm{Cr})$, and cobalt $(\mathrm{Co})$. In vitro studies demonstrated toxic effects of $\mathrm{Cd}$ and $\mathrm{Cr}$ on bone cells. In particular, ions form of these elements affects osteoblast proliferation, size, and shape (Bhattacharyya, 2009; Soudani et al., 2011). Moreover, Cd and $\mathrm{Cr}$ are able to influence osteoblastic activity by decreasing alkaline phosphatase levels and calcium accretion (Dermience et al., 2015). Co is an essential trace element since it is the core component of vitamin B12. Several in vitro studies revealed that Co influences bone resorption and bone formation by modulating bone cell metabolism (Dermience et al., 2015). Moreover, Fleury et al. demonstrated that Co accumulation increases oxidative stress in bone tissues (Fleury et al., 2006). These effects could be mediated by the redox state in osteoblast-like cells, but the mechanism of action is not known (Fleury et al., 2006). However, studies were mainly performed in vitro or in human serum and rarely on bone by ultrastructure analysis. Therefore, the aim of this study was to investigate whether heavy metals accumulation in bone tissues, i.e. $\mathrm{Pb}, \mathrm{Cd}, \mathrm{Co}$, and $\mathrm{Cr}$, could be related to the altered bone metabolism and architecture of osteoporotic patients.

\section{MATERIAL END METHODS}

\section{Patients}

In this retrospective study, we enrolled 25 patients that underwent hip arthroplasty for medial femoral fracture (OP) (18 women and 7 men; age $77.80 \pm 1.94)$ and 25 patients underwent hip arthroplasty for osteoarthritis (15 women and 10 men; age 73.80 \pm 1.24 ) in the Orthopedic Department of "Tor Vergata" University. Moreover we enrolled 15 patients (7 women and 8 men; age 46.19 \pm 2.78 ) underwent hip arthroplasty for high-energy hip fracture of the femoral head as a control group. All patients enrolled in the study lived in the metropolitan area of Rome, Italy for at least 30 years.

Exclusion criteria were history of neoplastic diseases, myopathies or other neuromuscular diseases or chronic administration of corticosteroid for autoimmune diseases (more than 1 month), diabetes, alcohol abuse, viral chronic infections (HBV, HCV, HIV), previously orthopedic surgical implants.

\section{Bone Mineral Density Evaluation}

Bone mineral density evaluation (DXA) was performed with a Lunar DXA apparatus (GE Healthcare, Madison, WI). Lumbar spine (L1-L4) and femoral (neck and total) scans were performed, and bone mineral density (BMD) was analyzed according to manufacture recommendations (Celi et al., 2013). Dual-energy X-ray absorptiometry measures BMD (in grams per square centimeter) with a coefficient of variation of $0.7 \%$. For patients with cervical femoral fragility fracture, BMD was measured on the limb opposite to the fracture side. Conversely, for the other patients all measurements were performed on the nondominant side, while participants lay supine on an examination table with their limbs abducted away from the trunk (Piccirilli et al., 2014). DXA exam was performed 1 day before surgery for OA patients and 1 month after surgery for hip fracture patients. Results were expressed as $T$ scores values.

\section{Radiographic Analysis}

Hip radiographs of all patients were obtained using a standard, validated protocol (Kothari et al., 2004). Two orthopedists independently, and at different time, assessed all radiographs using the Kellgren-Lawrence $(\mathrm{K}-\mathrm{L})$ 


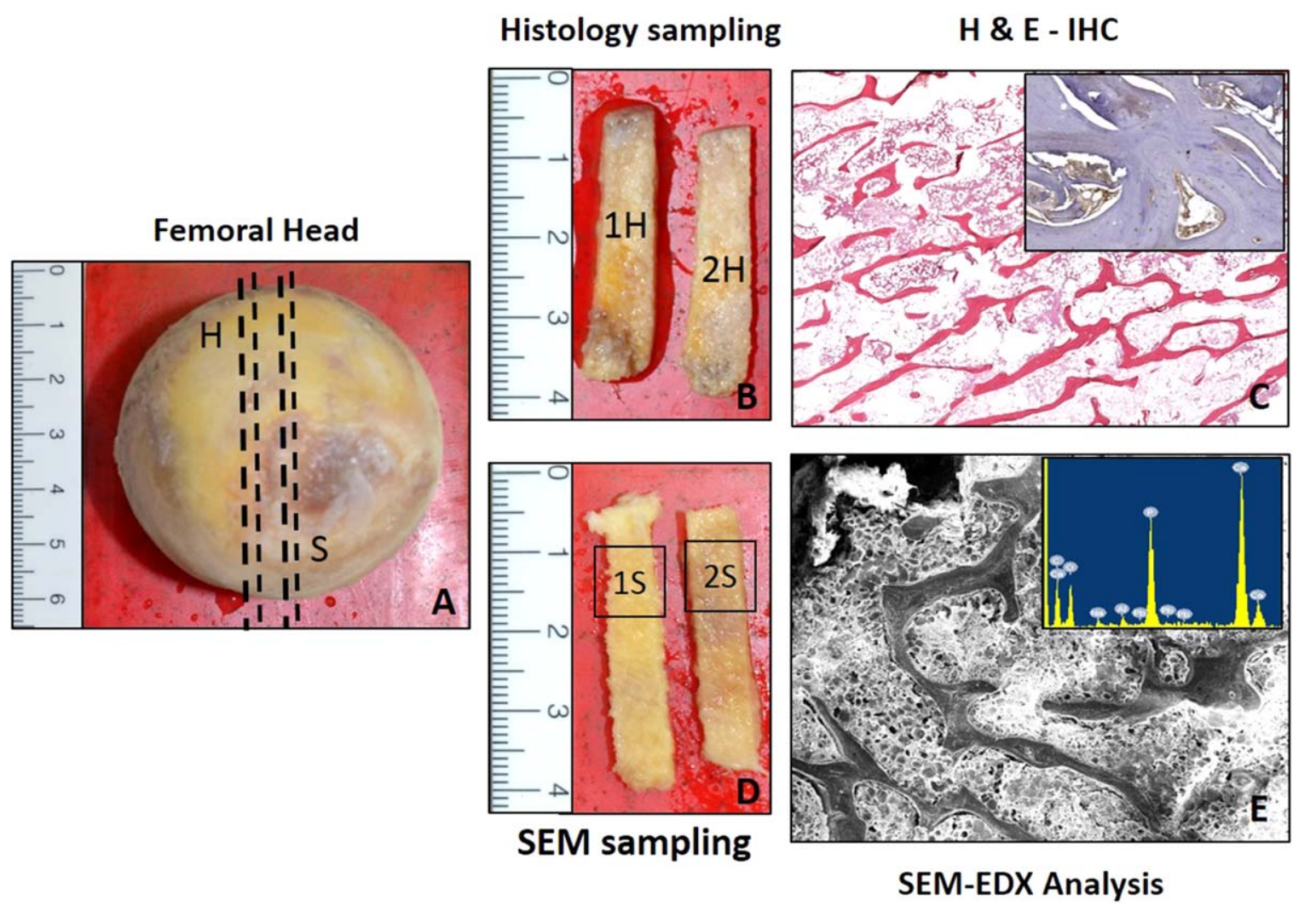

Fig. 1. Bone head sampling. (A) During open surgery of hip arthroplasty, we took the femoral head to obtain a bioptic sample. Subchondral bone columns of $0.8 \mathrm{~cm}$ in diameter and $3 \mathrm{~cm}$ in length were extracted from the loading area of the femoral head. (B, C) Two columns obtained by cutline "M" were fixed in formalin $4 \%$ and embedded in paraffin for histological and immunohistochemical analysis. (D, E) From two columns obtained by cutline "S" we dissected $0.8 \mathrm{~cm}$ in diameter and $1 \mathrm{~cm}$ in length of bone trabecular tissue distant $0.2 \mathrm{~cm}$ to cartilage for SEM-EDX studies. [Color figure can be viewed in the online issue, which is available at wileyonlinelibrary.com.]

radiographic atlas (Kellgren and Lawrence, 1957). Disagreements between the two orthopedists were resolved by consulting a third orthopedist. If at least one hip joint was graded as $\mathrm{K}-\mathrm{L} \geq 2$, the participant was diagnosed with radiographic hip osteoarthritis.

\section{Hematochemical Exam}

During hospitalization, we collected blood samples by both OP and OA patients.

Samples $(n=50)$ were investigated for serum concentrations assay of creatinine, azotemia (BUN), phosphorus, calcium, Vit D (25OHD3) and intact PTH.

\section{Sampling}

During open surgery of hip arthroplasty, we took the femoral head to obtain a bioptic sample (Fig. 1). For bioptic procedure, we excluded macroscopic alteration of trabecular bone as necrosis areas. All sampling and experiments were performed in agreement with independent ethical committee of "Policlinico Tor Vergata" (approval reference number \# 85/
12), and Informed consent was obtained from all individual participants included in the study.

\section{Histology and Histomorphometric Analysis}

Bone head samples were fixed in $4 \%$ paraformaldehyde for $24 \mathrm{~h}$ and paraffin embedded without decalcification process. From each patient, we obtained two paraffin blocks. Finally, 3 - $\mu$ m-thick sections were cut with tungsten carbide knife and stained with hematoxylin and eosin ( $\mathrm{H} \& \mathrm{E})$.

For each bioptic sample of OP and OA patients, we evaluated at least 10 microscopic images randomly selected from at least two bone slides for patient. Images were acquire at $40 \times$ magnification by using a Nikon Eclipse E600light microscope connected to a Nikon digital camera and saved at a resolution of $1280 \times 1024$ pixels. Successively, the images were analyzed by using a BioQuant Osteo software (version7.20.10; BIOQUANT Image Analysis Corporation, Nashville, USA) according to the manufacture's suggestions (Tarantino et al., 2014). From the BioQuant Osteo analysis we evaluated the following parameters: bone volume (BV/TV), trabecular thickness (Tb.Th) and trabecular separation (Tb.Sp). 
Washings were performed with PBS/Tween20 pH 7.6 (UCS diagnostic, Rome, Italy); reactions were revealed by HRP-DAB Detection Kit (UCS diagnostic, Rome, Italy).

\section{Scanning Electron Microscopy (SEM)}

One $\mathrm{cm}$ in diameter and $1 \mathrm{~cm}$ in length of bone trabecular biopsies, distant $0.2 \mathrm{~cm}$ to cartilage (Fig. 1), were fixed in $4 \%(\mathrm{v} / \mathrm{v})$ paraformaldehyde for $24 \mathrm{~h}$. After washing with $0.1 \mathrm{M}$ phosphate buffer, the sample was dehydrated by a series of incubations in 30,50, and 70\% (v/v) ethanol. Dehydration was continued by incubation steps in $95 \%$ (v/v) ethanol, absolute ethanol and acetone. Critical point drying (Agar Scientific, Essex, UK, Elektron Technology UK, Cambridge, UK) with supercritical $\mathrm{CO}_{2}$ was then performed to prevent cell deformation (Carpentieri et al., 2015). A SEM LEO 1450VP (Carl Zeiss Meditec, Oberkochen, Germany) was employed to acquire backscattered electron images using $20 \mathrm{keV}$ electrons leading to an information depth of about $1.5 \mu \mathrm{m}$. Images with a scan size of $30 \times 30$ $\mu \mathrm{m}^{2}$ were acquired, at a resolution of $1024 \times 1024$ pixels.

\section{EDX Microanalysis}

In recent studies, we applied EDX assay to human biopsies to study both the elemental composition of tissue and heavy metals associated to lung cancers (Scimeca et al. 2014a,b; Scimeca et al., 2016a).

The EDX microanalysis performs the chemical analysis of a sample describing the elemental composition of a tissue utilizing electron microscopy techniques. When the electron beam in an electron microscope hits a thin sample, some atoms of the sample will be excited or ionized. When they return into their ground state, they will emit characteristic Xrays (Scimeca et al., 2016b). The X-ray emission at different wavelengths may then be measured by a photon-energysensitive detector. EDX microanalysis was performed on unpost-fixed and uncoated bone biopsies were placed on specific stub using a liquid N2-cooled Si detector with a superultrathin Be window. Spectra were collected by a SEM LEO $1450 \mathrm{VP}$ scanning electron microscope at acceleration voltage of $5 \mathrm{KeV}$ employing an area scan mode $(640 \times 640$ $\mu \mathrm{m}^{2}$ sampling area), $300 \mathrm{~s}$ acquisition time, and $32-37 \%$ detector dead time. Analysis of acquired spectra was performed under a nonstandard mode using atomic numberabsorption-florescence correction (ZAF) methods using Inca Energy software (Oxford Instruments, High Wycombe, UK; $\mathrm{Si}(\mathrm{Li})$ detector, ATW - atmospheric thin window, resolution $133 \mathrm{eV}$ for $\mathrm{Mn} \mathrm{K} \alpha$ at 10,000 counts). For each specimen, we acquire five spectrum on $8 \mathrm{~mm}^{2}$ of bone surface in total. Results were expressed as presence or absence of metals.

\section{Immunohistochemistry}

Immunohistochemical characterization was performed to assess sclerostin expression in all femoral head biopsies.
Briefly, 3- $\mu$ m-thick sections were pre-treated with EDTA citrate $\mathrm{pH} 7.8$ for $30 \mathrm{~min}$ at $95^{\circ} \mathrm{C}$ and then incubated with mouse monoclonal Anti-sclerostin antibody for $60 \mathrm{~min}$ (1:100, clone MY-32, AbCam).

\section{Statistical Analysis}

Descriptive statistics was used to summarize pertinent study information. Categorical variables were reported as frequencies and percentage values, whereas continuous variables were summarized by mean values and their relative standard deviations. Correlations between categorical variables were evaluated through non-parametric tests, such as the Pearson's Chi-Square test or the Fisher exact test, when appropriate. $P$ values $<0.05$ denote statistically significant correlations. Statistical analyses were carried out using the SPSS software (SPSS ver. 21.0, SPSS, Chicago, IL). Clinical and Immunohistochemical data were analyzed by MannWhitney test $(p<0.0005)$ using Graph Pad Prism 5 Software (La Jolla, CA).

\section{RESULTS}

\section{Histomorphometrical Analysis}

The BioQuant Osteo software allowed us to analyze the bone quality parameters such as bone volume (BV/TV), trabecular thickness (Tb.Th), and trabecular separations (Tb.S) [Fig. 2(A-C)]. As regards to BV/TV, we observed a significantly higher mean value in $\mathrm{OA}$ patients respect to OP group (OA $0.251 \pm 0.018$ vs. OP $0.113 \pm 0.008 \mathrm{~mm}^{2}$ ) [Fig. 2(A)]. Noteworthy, dispersive graph shows that OA group was characterized by very heterogeneous BV/TV values. Indeed, in this group we observed patients with both high value of $\mathrm{BV} / \mathrm{TV}$ and levels of BV/TV compatible with OP condition. However, our data indicated that in OA group low BV/TV values were associated with older patients. Tb.Th values were significantly higher in OA respect to OP groups (OA $0.242 \pm 0.084 \mathrm{~mm}$ vs. OP $0.112 \pm 0.027 \mathrm{~mm}$ ) [Fig. 2(B)]. In OP patients, Tb.Th values were very low and only six patients were characterized by values similar to those of OA group. Conversely, we note a homogenous distribution of Tb.Th values in OA. As concern Tb.S values OP patients showed higher values respect to OA [Fig. 2(C)] (OA $0.499 \pm 0.115 \mathrm{~mm}$; OP $1.080 \pm 0.269 \mathrm{~mm}$ ).

\section{Heavy Metals Detection}

In this study we set up an ultrastructural microanalysis assay in order to characterize the presence of $\mathrm{Pb}, \mathrm{Cr}, \mathrm{Cd}$, and $\mathrm{Co}$ in head femoral biopsies [Fig. 2(D-F)]. Differences between OP and CTRL and OA groups were statistically significant in terms of $\mathrm{Pb}, \mathrm{Cr}$, and $\mathrm{Cd}$ accumulation in bone (Fig. 3). Considering the individual elements, $\mathrm{Pb}$ was detected in 92\% of $\mathrm{OP}$ samples, whereas $\mathrm{Cr}$ and $\mathrm{Cd}$ were present in 

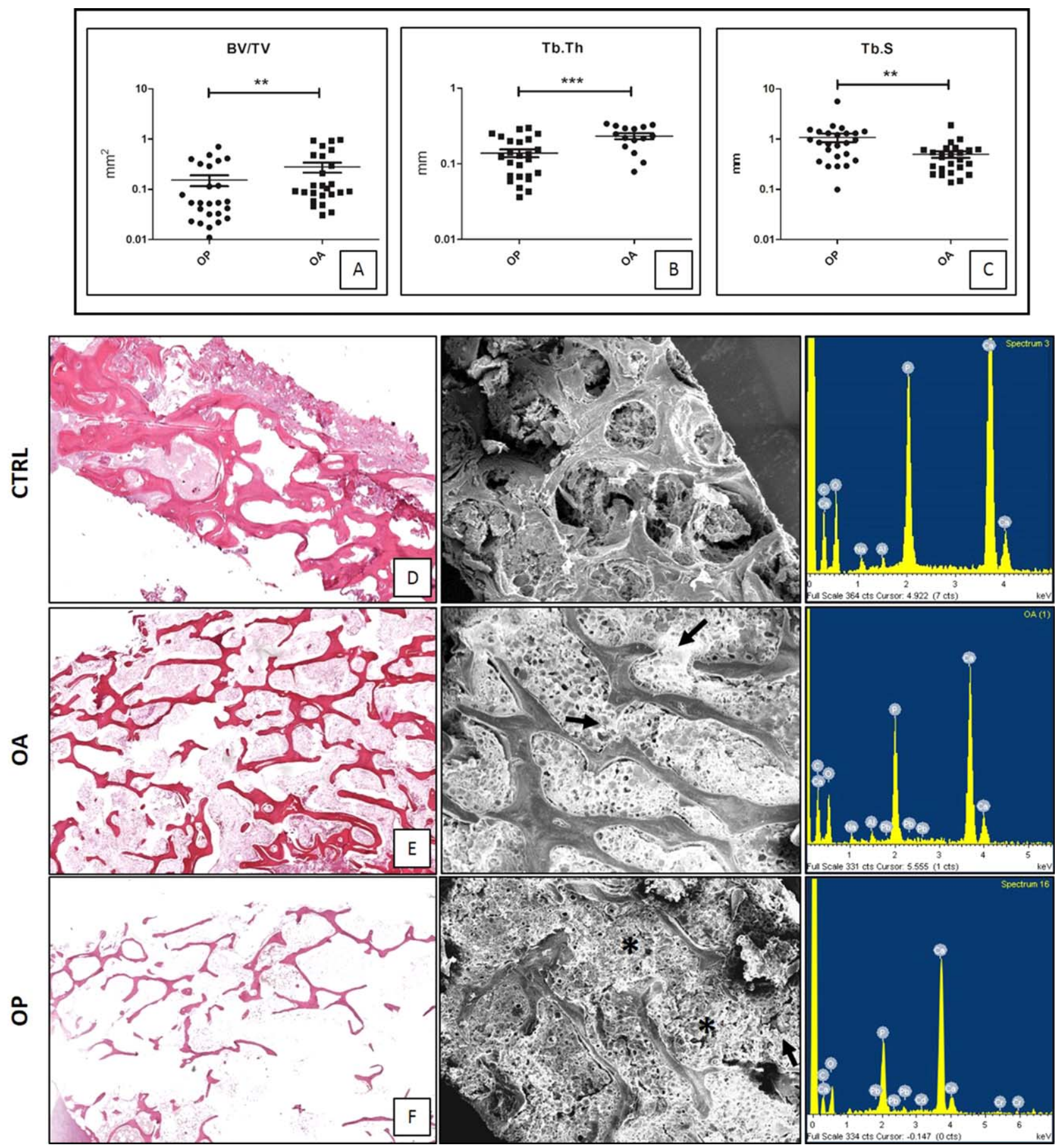

Fig. 2. Evaluation of bone morphometric parameters and SEM-EDX microanalysis. The BioQuant Osteo software allowed us to analyze the bone quality parameters such as Bone Volume (BV/TV), trabecular thickness (Tb.Th) and trabecular separations (Tb.S) of OP and OA patients $(A-C)$. (A) Graph show a significantly higher mean value of BV/TV in OA patients respect to OP group. (B) In OP patients, Tb.Th values were very low and only six patients were characterized by values similar to those of OA group. Conversely, we note a homogenous distribution of Tb.Th values in OA. (C) Tb.S values were significantly higher in OP patients compare to OA. Morphological analysis and heavy metal detection (D-F). (D) CTRL patients were characterized by a conserved trabecular net $(H \& E \times 2$; electron micrograph $\times 60$ ). (E) Bone head biopsies of OA patients display moderate trabecular thinning and decrease of transversal trabeculae (arrows) (H\& E $\times 2$; electron micrograph $\times 60$ ). (F) OP patients showed a remarkable loss of both trabecular thickness and number of transversal trabeculae $(H \& E \times 2$; electron micrograph $\times 60)$. Bone marrows was frequently substituted by fat (asterisks). [Color figure can be viewed in the online issue, which is available at wileyonlinelibrary.com.] 

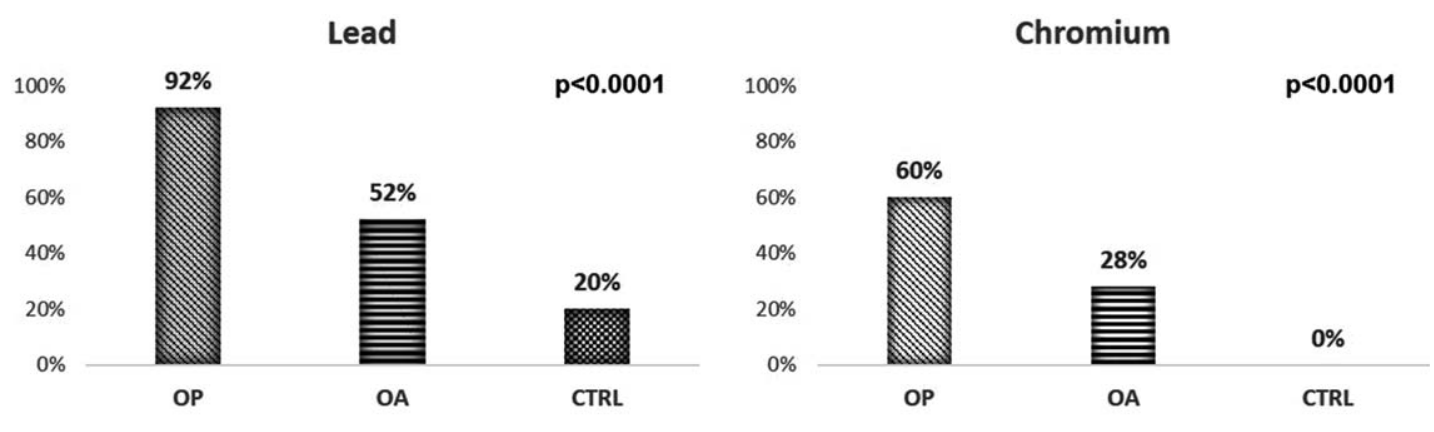

Cadmium
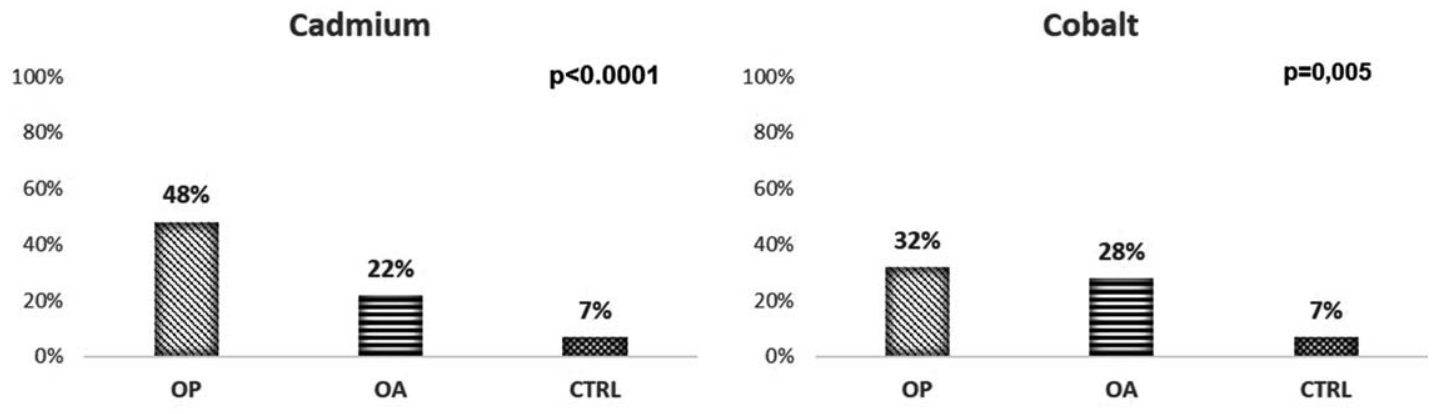

At last 2 elements
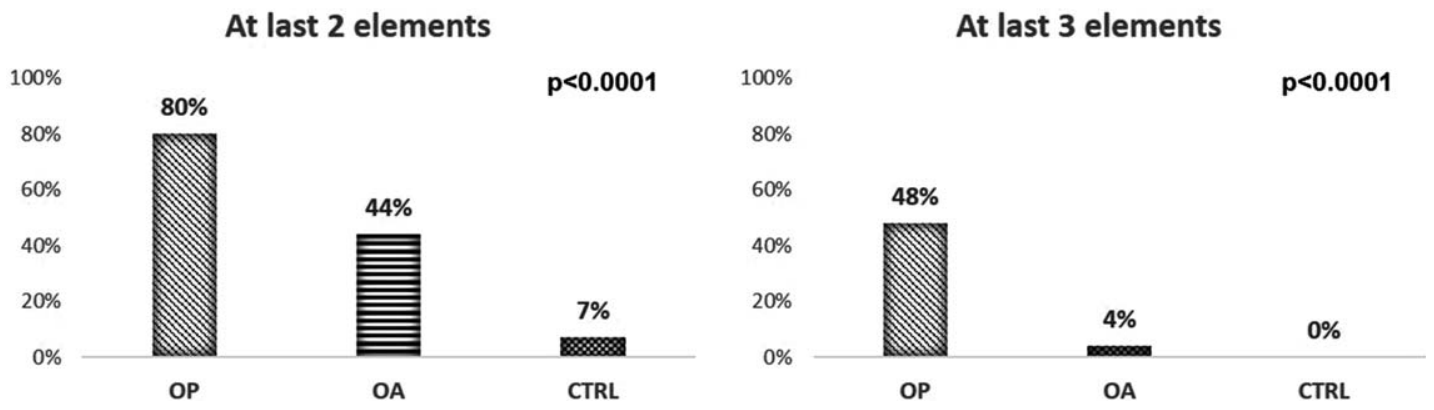

Fig. 3. Heavy metals accumulation in osteoporotic patients. Percentage distributions of the four heavy metals according to the three group of tissues: (A) lead (Pb); (B): chromium (Cr); (C): cadmium (Cd); (D): cobalt (Co). The presence of heavy metals revealed respectively in OP, OA and CTRL were reported as percentage. ${ }^{*}$ Chi-Square's test. $P$ values refer to comparisons between the two groups of tissues according to the presence of each specific element.

$60 \%$ and $32 \%$, respectively (Fig. 3). Conversely, we did not found differences in the accumulation of Co (OP 32\%; OA, 28\%; CTRL 7\%) (Fig. 3). A high rate of OP femoral head biopsies showed the co-presence of more than one bioaccumulated element (Fig. 3). Interestingly, we could find significant differences $(P<0.0001)$ among the groups, when we compared the bio-accumulation of two or more elements. In particular, at least two elements were present in $80 \%$ of OP, $44 \%$ of OA, and $7 \%$ CTRL, whereas more than three elements were found in $48 \%$ of OP and $4 \%$ OA (Fig. 3). The frequency of accumulation of toxic elements in CTRL group was less and the presence of two elements was only occasionally reported (at least two elements 7\%; at least three elements 0\%) (Fig. 3).

\section{Immunohistochemistry}

Sclerostin analysis was performed by immunohistochemistry and the levels of its expression were quantified by assigning a score from 1 to 3 according to the intensity of positive signals in osteocyte cells. As aspect, we observed a significantly higher signal of sclerostin in OP group respect to both CTRL and OA patients (OP $2.28 \pm 0.15$; OA $1.32 \pm 0.13$; CTRL $0.92 \pm 0.08$ ) (fig.4). We also compared the expression of sclerostin between OP patients with at least three heavy metal elements and OP patients with less than three elements. Our data showed a significant rise of sclerostin expression in patients with at least three elements [Fig. 4(H)]. Surprisingly, only in OP patients, sclerostin was expressed by bone marrow adipocytes too.

\section{Clinical Evaluation}

Clinical and instrumental evaluation allowed us to characterize patients enrolled in this study. OP group included 25 patients with fragility hip fracture, $T$ score $\leq-2.5 \mathrm{SD}$ and $\mathrm{K}-\mathrm{L}$ score from 0 to 1 ; whereas $\mathrm{OA}$ group included 25 patients with positive radiogram for hip OA with a $\mathrm{K}-\mathrm{L}$ 

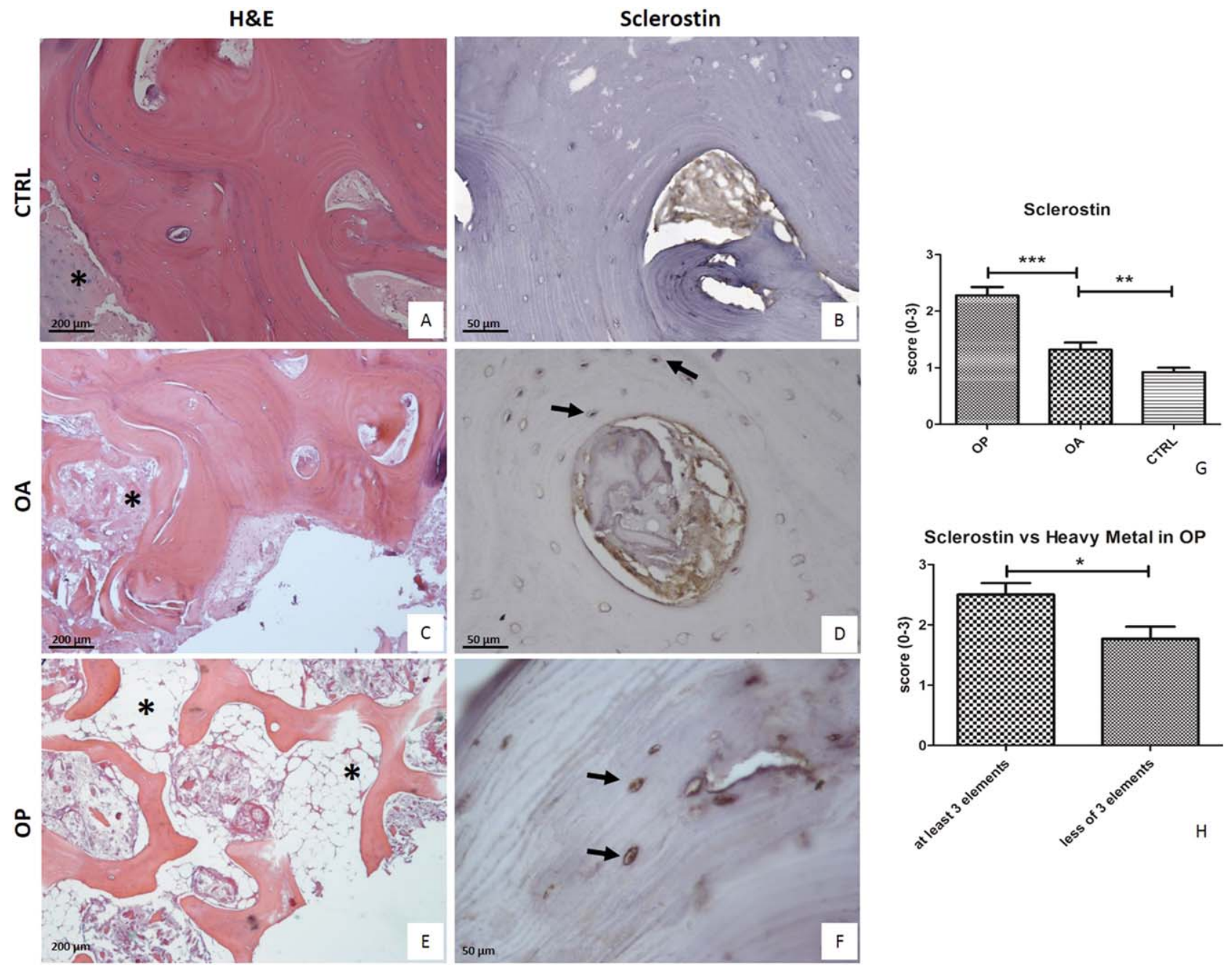

Sclerostin vs Heavy Metal in OP

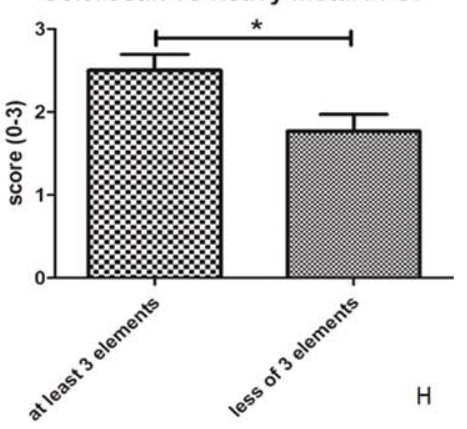

Fig. 4. Expression of sclerostin in bone head biopsies. (A) H \& E section showed a bone tissue of CTRL group with a conserved trabecular net and remodeling area (asterisk) $(\times 4)$. (B) Image displayed no/low expression of sclerostin in a CTRL patient. (C) H \& E section of OA bone tissues $(\times 4)$. Bone biopsies are characterize by compact trabeculae. (D) Immunohistochemical analysis show a lower/moderate expression of sclerostin by osteocytes (arrows) (X10). (E) H \& E section show a bone tissue of OP patients $(\times 4)$. Trabeculae were thinned and bone marrows was frequently substituted by fat (asterisks) $(F)$ Immunohistochemical reaction display high sclerostin expression by osteocyte cells (arrows) (×10). (G) Graph showed a significantly higher signal of sclerostin in OP group respect to both OA and CTRL patients. (H) Graph displayed a significant rise of sclerostin expression in OP patients with at least three elements respect to OP patients with less than three elements. [Color figure can be viewed in the online issue, which is available at wileyonlinelibrary.com.]

score 3 or 4 and $T$ score $\geq-2.5$ SD. Moreover, OA and OP patients displayed homogenous values of mainly hematochemical exams of both bone and kidney metabolism (Table I). Finally, for CTRL patients we assume $T$ score $\geq-2.5 \mathrm{SD}$ and $\mathrm{K}-\mathrm{L}$ score from 0 to 1.

\section{DISCUSSION}

The skeleton is a metabolically active organ since mechanical, genetic and environmental factors affect bone metabolisms that undergoe continuous remodeling throughout life. Nevertheless, the role of environmental pollution on bone metabolism is still unclear and controversial. Exposure to heavy metals, the most important pollutants capable to affect bone mass (Berglund et al., 2000; Sadeghi et al. 2014; Zheng et al., 2014; Tsai et al., 2015 ) continues even though several adverse health effects have been known and international associations such as the WHO (WHO, 1992, 1995) monitor them regularly. The main threats to human health from heavy metals are associated with $\mathrm{Pb}, \mathrm{Cd}, \mathrm{Co}$, and $\mathrm{Cr}$ exposure.

To investigate the influence of $\mathrm{Pb}, \mathrm{Cr}, \mathrm{Cd}$, and $\mathrm{Co}$ on a metabolic disorder of the bone disease such as osteoporosis, we analyzed 25 bone biopsies of OP patients with femur fragility fracture by SEM-EDX microanalysis to evaluate their 
TABLE I. Main anamnestic and instrumental data of OP, OA, and CTRL patients

\begin{tabular}{|c|c|c|c|c|}
\hline & & & & $T$ Test \\
\hline & OP & $\mathbf{O A}$ & CTRL & (Mann-Whitney Test) \\
\hline$\overline{\text { Age }}$ & $77.80 \pm 1.94$ & $73.32 \pm 1.24$ & $46.19 \pm 2.78$ & $\begin{array}{c}\text { OP vs OA ns }(P=0.13 ; \text { OP vs } \\
\text { CTRL } * * *(P<0.001) ; \text { OA vs } \\
\text { CTRL } * * *(P<0.001)\end{array}$ \\
\hline BMI & $22.58 \pm 1,50$ & $28.06 \pm 0.80$ & / & $* * *(P=0.0005)$ \\
\hline T Score (L1-L4) & $-2.8 \pm 0.17$ & $0.45 \pm 0.5$ & / & $* * *(P=0.0005)$ \\
\hline T Score (neck) & $-2.7 \pm 0.28$ & $-0,37 \pm 0.33$ & / & $* * *(P=0.0005)$ \\
\hline Creatinine (BUN) $\left(\mathrm{mg} \mathrm{dL}^{-1}\right)$ & $0.97 \pm 0.10$ & $1.5 \pm 0.34$ & / & ns $(P=0.15)$ \\
\hline Azotemia $\left(\mathrm{mg} \mathrm{dL}^{-1}\right)$ & $48.80 \pm 4.7$ & $49.6 \pm 6.00$ & / & ns $(P=0.60)$ \\
\hline Phosphorus (mg dL ${ }^{-1}$ ) & $2.92 \pm 0.08$ & $3.52 \pm 0.21$ & / & $* *(P=0.0083)$ \\
\hline Calcium $\left(\mathrm{mg} \mathrm{dL}^{-1}\right)$ & $8.45 \pm 0.12$ & $8.60 \pm 0.11$ & / & $\mathrm{ns}(P=0.29)$ \\
\hline Vit D(25OHD3) (ng mL $\mathrm{m}^{-1}$ ) & $10.26 \pm 2.60$ & $21.63 \pm 9.00$ & / & ns $(P=0.47)$ \\
\hline PTH $\left(\right.$ pg mL $\left.\mathbf{~ L}^{-1}\right)$ & $135.10 \pm 41.00$ & $81.00 \pm 10.00$ & / & $\mathrm{ns}(P=0.62)$ \\
\hline
\end{tabular}

elemental composition. In addition, we enrolled OA patients to compare heavy metal accumulation in patients with similar age range. As control group, we studied bone femoral head biopsies of patients with high-energy fracture or osteonecrosis (CTRL).

Bone morphometric analysis showed a reduction of both $\mathrm{BV} / \mathrm{TV}$ and Tb.Th in OP patients. Moreover, in this group, bone remodeling was often missing (data not shown). Nevertheless, evaluating the distribution of bone quality values, we noted that some OP patients showed bone quality comparable to OA group. The evaluation of bone morphology allowed us to demonstrate that cells involved in bone remodeling (osteoblasts, osteoclasts, and osteocytes) were often rare or absent in bone head trabecular tissues of OP patients.

Several studies demonstrated the epidemiological correlation between environmental pollution and occurrence of OP (Kung and Huang, 2007; Ongphiphadhanakul, 2007). However, to the best of our knowledge, any study has been performed about heavy metals accumulation into bone tissue at the ultrastructural and microanalytical level.

Thanks to the SEM-EDX microanalysis we demonstrated a specific accumulation of heavy metals in trabecular bone tissue. Toxic elements bio-accumulation appears a time dependent phenomenon, as demonstrated by the low presence of metals in under 50 patients (CTRL). Indeed, we demonstrated that trabecular tissues of OP patients harbor a higher number heavy metals (i.e., $\mathrm{Pb}, \mathrm{Cr}$, and $\mathrm{Cd}$ ) than patients with comparable age (OA). It should be noted that the heavy metals we detected coincide with those emitted in the atmosphere by natural and/or anthropic sources (Bell et al., 2009; Srimuruganandam and Nagendra, 2011).

The most frequent element in bone of OP group was $\mathrm{Pb}$. In vitro experiments demonstrated the ability of $\mathrm{Pb}$ to inhibit osteoblast differentiation and activity (Dermience et al., 2015). In particular, it affects hormonal secretion and hormonal-induced cell responses, particularly to 1, $\alpha 25$-dihydroxycholecalciferol and IGF-1, inhibits bone-related protein synthesis, including osteocalcin, collagen and osteopontin, and suppresses Wnt signaling (Angle et al., 1990, Sauk et al., 1992). The possible inhibition of osteoblast exerted by $\mathrm{Pb}$, allowed us to explain the inverse correlation between $\mathrm{Pb}$ accumulation and bone quality observed in our samples. Indeed, we frequently detected $\mathrm{Pb}$ in bone tissues of $\mathrm{OP}$ patients with lower values of BV/TV (data not shown). Noteworthy, the presence of $\mathrm{Pb}$ was observed also in $52 \%$ of OA patients and in $20 \%$ of CTRLs. For long time the negative effect of $\mathrm{Cd}$ on bone mass was attributed to its capability to cause renal tubular dysfunction (Schutte et al., 2008). However, recent studies highlighted the direct role of cadmium on bone metabolisms (Yokota and Tonami, 2008). The direct pathway implicated in cadmium bone toxicity could be linked to calcium homeostasis and bone cell metabolism. Because of similar properties, Cd can impair calcium metabolism during osteogenesis and bone homeostasis by increasing calciuria and altering calciotropic hormones (Dermience et al., 2015). In this context, our data support the hypothesis of a direct role of $\mathrm{Cd}$ in bone metabolism. Indeed, as for $\mathrm{Pb}$, we displayed $\mathrm{Cd}$ accumulation in patients with lower bone quality parameters. Moreover, tissues with both $\mathrm{Pb}$ and $\mathrm{Cd}$ accumulation were characterized by both poor bone quality and reduction/absence of osteoblasts. $\mathrm{Cr}$ intoxication from environmental exposure is not common, except in chromium-related industries and associated environments (Soudani et al., 2011). Reduced bone formation may be mediated via cytotoxic effects of $\mathrm{Cr}$ on osteoblasts, which reduce proliferation, inhibit osteocalcin release, and decrease alkaline phosphatase activity. These effects are associated with oxidative stress, which can be caused by unbalanced OPG/RANKL ratios and dysregulation of antioxidant enzyme expression (Anderson et al., 1997). Our data showed that the accumulation of $\mathrm{Cr}$ into bone structure was less frequent respect to $\mathrm{Pb}$. However, in some $\mathrm{OP}$ samples we identified simultaneous presence of $\mathrm{Pb}, \mathrm{Cd}$, and $\mathrm{Cr}$. Noteworthy, in the context of OP group we found a significantly 
difference between $t$ score values of patients with at least three elements $(\mathrm{Pb}$ included) and $t$ score values of patients with less than three elements. In particular, OP patients with at least three elements showed a low BMD. Moreover, these patients showed low values of main morphological parameters such as BV/TV and Tb.Th (data not shown). In the human, the inverse relationship between heavy metals $(\mathrm{Pb}$ and $\mathrm{Cd}$ ) and BMD values were already described (Khalil et al., 2008; Callan et al., 2015; Rzymski et al., 2015; García-Esquinas et al., 2015). Nevertheless, in these studies heavy metals were found in blood but not into bone structure. Therefore, the ability of heavy metals to affect BMD could be just speculate. Indeed, the accumulation of heavy metals in the blood can indirectly influence bone metabolism affecting renal function and/or female reproductive system (Rzymski et al., 2015).

To correlate heavy metal accumulation with bone metabolism, we studied the expression of sclerostin, a protein with anti-anabolic effects on bone formation (Yavropoulou et al., 2014). High levels of sclerostin revealed in tissues with $\mathrm{Pb}$, $\mathrm{Cd}$, and $\mathrm{Cr}$, candidate it as a molecular link between heavy metals accumulation and bone metabolism impairment. Indeed, sclerostin expression was higher in tissues with at least two heavy metals accumulated.

Summing up, our data highlighted the importance of $\mathrm{Pb}$, $\mathrm{Cd}$, and $\mathrm{Cr}$ in bone health. The presence of these elements is convincingly connected to the environmental pollution, as assessed by habitat conditions of the investigated patients. Indeed, they live in a large urban center in which heavy metals concentration is often upper safety threshold values. However, the possible sources of heavy metals, such as contaminated water and food, dental implant, etc., are numerous and difficult to identify.

\section{CONCLUSION}

The identification of heavy metals into bone structure opens new perspectives about the pathogenesis of osteoporosis. In addition, from clinical point of view, the detection of heavy metals contamination into the bone or other tissues (i.e., blood) could become a powerful tool to assessing bone status. To better elucidate the molecular mechanism in which heavy metals can participate to osteoporosis development in humans, in vivo and in vitro studies are needed. Furthermore, it would be interesting to validate the results of present study in a larger cohort of patients correlating SEM-EDX data with serum concentration of heavy metals. In fact, heavy metals, such as $\mathrm{Pb}, \mathrm{Cr}$, and $\mathrm{Cd}$, could contribute to development of osteoporosis at both cellular/molecular and epigenetic level.

It was very important to take advantage of the funds made available by the ASI because it encourages our present and further research within the project titled "Multidisciplinary Study of the
Effects of Microgravity on Bone Cells" call number search DCDTE-2011-033.

\section{REFERENCES}

Anderson DM, Maraskovsky E, Billingsley WL, Dougall WC, Tometsko ME, Roux ER, Teepe MC, DuBose RF, Cosman D, Galibert L. 1997. A homologue of the TNF receptor and its ligand enhance T-cell growth and dendritic-cell function. Nature 390:175-179.

Andreoli A, Bazzocchi A, Celi M, Lauro D, Sorge R, Tarantino U, Guglielmi G. 2011. Relationship between body composition, body mass index and bone mineral density in a large population of normal, osteopenic and osteoporotic women. Radiol Med 116:1115-1123.

Angle CR, Thomas DJ, Swanson SA. 1990. Lead inhibits the basal and stimulated responses of a rat osteblast- like cell line ROS $17 / 2.8$ to 1,25 dihydroxyviatmin D3 and IGF-1. Toxicol Appl Pharmacol 103:281-287.

Bell ML, Ebisu K, Peng RD, Dominici F. 2009. Adverse health effects of particulate air pollution: Modification by air conditioning. Epidemiology 20:682-686.

Berglund M, Akesson A, Bjellerup P, Vahter M. 2000. Metalbone interactions. Toxicol Lett 15:219-225; 112-113.

Bhattacharyya MH. 2009. Cadmium osteotoxicity in experimental animals: Mechanisms and relationship to human exposures. Toxicol Appl Pharmacol 238:258-265.

Callan AC, Devine A, Qi L, Ng JC, Hinwood AL. 2015. Investigation of the relationship between low environmental exposure to metals and bone mineral density, bone resorption and renal function. Available at: http://dx.doi.org/10.1016/j.ijheh.2015. 03.010

Carpentieri A, Cozzoli E, Scimeca M, Bonanno E, Sardanelli AM, Gambacurta A. 2015. Differentiation of human neuroblastoma cells toward the osteogenic lineage by mTOR inhibitor. Cell Death Dis 12:e1974.

Celi M, Rao C, Scialdoni A, Tempesta V, Gasbarra E, Pistillo P, Tarantino U. 2013. Bone mineral density evaluation in osteoporosis: Why yes and why not? Aging Clin Exp Res 25(Suppl 1): S47-S49.

Cerocchi I, Ghera S, Gasbarra E, Scialdoni A, Tarantino U. 2013. Fragility fractures: The clinical pathway. Aging Clin Exp Res 25:43-45.

Dermience M, Lognay G, Mathieu F, Goyens P. 2015. Effects of thirty elements on bone metabolism. J Trace Elem Med Biol 32:86-106.

Drake MT, Clarke BL, Lewiecki EM. 2015. The pathophysiology and treatment of osteoporosis. Clin Ther 37:1837-1850.

Fleury C, Petit A, Mwale F, Antoniou J, Zukor DJ, Tabrizian M, Huk OL. 2006. Effect of cobalt and chromium ions on human MG-63 osteoblasts in vitro: Morphology, cytotoxicity, and oxidative stress. Biomaterials 27:3351-3360.

García-Esquinas E, Navas-Acien A, Pérez-Gómez B, Artalejo FR. 2015. Association of lead and cadmium exposure with frailty in US older adults. Environ Res 137:424-431. 
James KA, Meliker JR. 2013. Environmental cadmium exposure and osteoporosis: a review. Int J Public Health 58:737-745.

Kellgren JH, Lawrence JS. 1957. Radiological assessment of osteoarthrosis. Ann Rheum Dis 16:494-502. doi: 10.1136/ ard.16.4.494.

Khalil N, Cauley JA, Wilson JW, Talbott EO, Morrow L, Hochberg MC, Hillier TA, Muldoon SB, Cummings SR. 2008. Relationship of blood lead levels to incident non spine fractures and falls in older women: The study of osteoporotic fractures. J Bone Miner Res 23:1417-1425.

Kothari M, Guermazi A, von Ingersleben G, Miaux Y, Sieffert M, Block JE, Stevens R, Peterfy CG. 2004. Fixed-flexion radiography of the knee provides reproducible joint space width measurements in osteoarthritis. Eur Radiol 14:1568-1573.

Kung AW I, Huang QY. 2007. Genetic and environmental determinants of osteoporosis. J Musculoskelet Neuronal Interact 7: 26-32.

O'Flaherty EJ. 2000. Modeling normal aging bone loss, with consideration of bone loss in osteoporosis. Toxicol Sci 55:171188.

Ongphiphadhanakul B. 2007. Osteoporosis: The role of genetics and the environment. Forum Nutr 60:158-167.

Piccirilli E, Gasbarra E, Baldi J, Pistillo E, Tarantino U. 2014. Can muscular impairment be the "key" for femoral fracture? J Gerontol Geriatr Res. 3:5 doi:10.4172/2167-7182.1000183.

Rzymski P, Tomczyk K, Rzymski P, Poniedziałek B, Opala T, Wilczak M. 2015. Impact of heavy metals on the female reproductive system. Ann Agric Environ Med 22:259-264.

Sadeghi N, Oveisi MR, Jannat B, Hajimahmoodi M, Behzad M, Behfar A, Sadeghi F, Saadatmand S. 2014. The relationship between bone health and plasma zinc, copper lead and cadmium concentration in osteoporotic women. J Environ Health Sci Eng 12:125.

Sauk JJ, Smith T, Silbergeld EK, Fowler BA, Somerman MJ. 1992. Lead inhibits secretion of osteonectin/SPARC without significantly altering collagen or $\mathrm{Hsp} 47$ production in osteoblast-like ROS 17/2.8 cells. Toxicol Appl Pharmacol 116: 240-247.

Schutte R, Nawrot TS, Richart T, Thijs L, Vanderschueren D, Kuznetsova T, Van Hecke E, Roels HA, Staessen JA. 2008. Bone resorption and environmental exposure to cadmium in women: A population study. Environ Health Perspect 116:777783.
Scimeca M, Bischetti S, Bonanno E. 2016a. Energy dispersive Xray (EDX) microanalysis in biomedical research. Lett Health Biol Sci 1:1-2.

Scimeca M, Giannini E, Antonacci C, Pistolese CA, Spagnoli LG, Bonanno E. 2014. Microcalcifications in breast cancer: An active phenomenon mediated by epithelial cells with mesenchymal characteristics. BMC Cancer 23:286.

Scimeca M, Orlandi A, Terrenato I, Bischetti S, Bonanno E. 2014. Assessment of metal contaminants in non-small cell lung cancer by EDX microanalysis. Eur J Histochem 1258:2403.

Scimeca M, Pietroiusti A, Milano F, Anemona L, Orlandi A, Marsella LT, Bonanno E. 2016b. Elemental analysis of histological specimens: A method to unmask nano asbestos fibers. Available at: http://dx.doi.org/10.4081/ejh.2016.2573

Soudani N, Ibtissem Ben A, Troudi A, Bouaziz H, Boudawara T, Zeghal N. 2011. Oxidative stress induced by chromium (VI) in bone of suckling rats. Toxicol Ind Health 27:724-734.

Srimuruganandam B, Nagendra SM. 2011. Chemical characterization of PM10 and PM2.5 mass concentrations emitted by heterogeneous traffic. Sci Total Environ 409:3144-3157.

Tarantino U, Celi M, Rao C, Feola M, Cerocchi I, Gasbarra E, Ferlosio A, Orlandi A. 2014. Hip osteoarthritis and osteoporosis: Clinical and histomorphometric considerations. Int J Endocrinol. 2014:372021. doi: 10.1155/2014/372021.

Tsai TL, Pan WH, Chung YT, Wu TN, Tseng YC, Liou SH, Wang SL. 2015. Association between urinary lead and bone health in a general population from Taiwan. J Expo Sci Environ Epidemiol. Jul:8. doi: 10.1038/jes.2015.30.

WHO. 1992. Cadmium. Environmental Health Criteria, Vol. 134. Geneva: World Health Organization.

WHO. 1995. Lead. Environmental Health Criteria, Vol. 165. Geneva: World Health Organization.

Yavropoulou MP, Xygonakis C, Lolou M, Karadimou F, Yovos JG. 2014. The sclerostin story: From human genetics to the development of novel anabolic treatment for osteoporosis. Hormones (Athens) 13:323-337.

Yokota H, Tonami H. 2008. Experimental studies on the bone metabolism of male rats chronically exposed to cadmium intoxication using dual-energy X-ray absorptiometry. Toxicol Ind Health 24:161-170.

Zheng J, Mao X, Ling J, He Q, Quan J. 2014. Low serum levels of zinc, copper, and iron as risk factors for osteoporosis: A metaanalysis. Biol Trace Elem Res 160:15-23. 